Machowiec Piotr, Maksymowicz Marcela, Niemirski Dominik. Analysis of the safety of using e-cigarettes during pregnancy. The literature review. Journal of Education, Health and Sport. 2020;10(9):793-802. eISSN 2391-8306. DOI http://dx.doi.org/10.12775/JEHS.2020.10.09.096

https://apcz.umk.pl/czasopisma/index.php/JEHS/article/view/JEHS.2020.10.09.096

https://zenodo.org/record/4052795

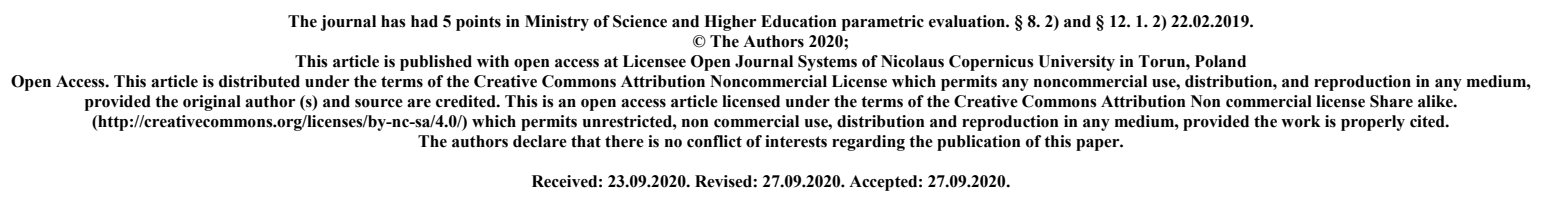

\title{
Analysis of the safety of using e-cigarettes during pregnancy. The literature review
}

\author{
Piotr Machowiec ${ }^{1}$, Marcela Maksymowicz ${ }^{1}$, Dominik Niemirski ${ }^{1}$ \\ ${ }^{1}$ Students' Scientific Association of Chair and Department of Epidemiology and Clinical \\ Research Methodology, Medical University of Lublin, Radziwiłłowska 11, 20-080 \\ Lublin, Poland \\ Corresponding author: Piotr Machowiec; piotr.machowiec1997@gmail.com \\ ORCID ID and E-mail: \\ Piotr Machowiec; https://orcid.org/0000-0002-5418-0110;piotr.machowiec1997@gmail.com \\ Marcela Maksymowicz; https://orcid.org/0000-0003-2611-1609;marcela.maksymowicz@gmail.com \\ Dominik Niemirski; https://orcid.org/0000-0003-1582-6975;dominikniemirski96@gmail.com
}

\begin{abstract}
Introduction and objective: The popularity of e-cigarettes around the world has been increasing in recent years. The reason for this may be a relatively lower cost of use and a lower amount of toxins compared to traditional cigarettes. E-cigarettes are more often picked by women of reproductive age compared to the general group of women.
\end{abstract}


Abbreviated description of the state of knowledge: According to recent studies, pregnant women consider e-cigarettes safer than tobacco cigarettes, which may be due to being advertised as a less harmful product compared to traditional cigarettes. Although some electronic cigarettes do not contain nicotine, they also consist of glycol, glycerin, fragrances and heavy metals that may adversely affect the development of the fetus. Furthermore, the statements of some significant healthcare institutions are divided on the safe use of ecigarettes by pregnant women, even in the case of treating them as an aid in the smoking cessation process.

Summary: No clear understanding is noticed regarding the effects of using electronic nicotine delivery systems during pregnancy on maternal, fetal or newborn health, so there is a high need of further studies assessing the safety of these noncombustible tobacco products. However, it can be an feasible alternative to traditional cigarettes, when trying to quit smoking by pregnant women.

Key words: e-cigarettes; pregnancy; safety; fetus

\section{Introduction and objective}

In recent years, new potential threats to public health have emerged, the so-called electronic cigarettes (e-cigarettes). They are perceived as less harmful, which is why many people find them especially attractive. E-cigarettes are electronic devices that heat a liquid and create an aerosol or mixture of small particles in the air. The e-liquid consists of: propylene glycol and/or glycerin, water or ethanol, nicotine (from 0 to $20 \mathrm{mg} / \mathrm{ml}$ ) and flavor additives.

Substances released from tobacco smoke are a risk factor for the development of many diseases - lung cancer, cervical cancer, COPD (chronic obstructive pulmonary disease), asthma or may contribute reduced fertility [1,2]. Exposing women during pregnancy to active and passive smoking increases the risk of preterm labor, IUGR, reduced lung function, and asthma in children $[1,3,4]$. On the other hand, quitting smoking reduces the occurrence of these complications. Due to the harmfulness of substances released from tobacco smoke, especially nicotine, on the health of the woman and the fetus, smoking is generally prohibited during pregnancy and breastfeeding. Nevertheless, some women continue to smoke during pregnancy. In the USA it is estimated that up to $20 \%$ of pregnant 
women proceed smoking, not only traditional but also various alternative forms of tobaccoderived products[5]. It is believed that this could be related to lower education level, having Medicaid coverage, white or Indian race, and young age.

In response to this phenomenon, the FDA (Food and Drug Administration) recommends that pregnant women should quit smoking, and if that is impossible, implement replacement therapy, even nicotine one [5]. However, complete cessation of smoking is difficult to achieve, so that e-cigarettes [6] are the most often chosen alternative by current and former smokers, which are often the option that helps women of reproductive age quit smoking $[7,8]$.

Pregnant women consider e-cigarette less harmful to health than traditional cigarettes. Nevertheless, the issue of the safety of their use by pregnant women has not been clearly defined, what requires an individual approach by doctors to pregnant patients who smoke, assessing benefits and drawbacks of e-cigarettes, both on women and fetus.

The search for clinical trials was based on a detailed protocol developed prior to the commencement of the systematic review paper. It took into account the criteria for including studies in the review, the search strategy, the method of selecting articles and the planned methodology for conducting data analysis. The following inclusion criteria were used: 1) studies published in English 2) available as full texts 3) published in the last 10 years. An analysis of scientific publications available on the PubMed and Google Scholar platform was carried out using the keywords linked by logical operators: "e-cigarette" and "pregnancy", as a result of which, after selection by two independent analysts, 20 scientific publications were used. The verification at the level of abstracts and titles was carried out in such a way that all reports considered useful by at least one of the analysts were included in the next stage.

The aim of the study was to present the current state of knowledge regarding the safety of using e-cigarettes during pregnancy with analysis of its potential complications in infants and older children.

\section{State of knowledge:}

\subsection{Epidemiology of e-cigarettes use.}

Depending on age, cigarettes are most popular among young adults, high school graduates, but e-cigarettes among younger adolescents and graduates [2,8]. In a study by Makadia et al., the majority of smokers started smoking before the age of 18 [5]. Starting 
smoking at this age may result in addiction in the reproductive age [9] and a greater tendency to use e-cigarettes in the future, also during pregnancy $[2,6]$. The popularity of ecigarettes is growing all over the world [10]. They are also more often used by women of reproductive age compared to the general group of women and they are a frequently chosen form of smoking cessation aid in pregnant women $[2,7,8,11]$. Other reasons for using ecigarettes include their safety, the taste of stimulants, a higher level of safety for a child, and the curiosity of using them [11,12]. Pregnant women consider e-cigarettes safer than traditional cigarettes, which may be due to being advertised as a less harmful product compared to traditional cigarettes. However, the consumption of e-cigarettes during pregnancy is criticized by others due to expectations of an impeccable lifestyle and sacrifice for the child by the mother [9].

However, it is estimated that from 0.6 to $15 \%$ of pregnant women smoke e-cigarettes, and according to other studies $9 \%$ of pregnant women smoke both traditional cigarettes and e-cigarettes [11]. The percentage of women using e-cigarettes is higher in the period of 2-6 months after giving birth than during the third trimester of pregnancy [12]. The tendency to consume them intensively may result from the mental and physical stress, associated with caring for the infant and many new tasks for the mother.

\subsection{Correlations associated with e-cigarette use.}

Lower education level, young age, white race and difficult financial situation are factors that increase the likelihood of smoking during pregnancy. According to Kurti et al. up to $40 \%$ of pregnant women smoking cigarettes concurrently use e-cigarettes [6]. The risk of starting e-cigarette use increases 28 times for current smokers compared to ex-smokers.

As Genna et al. also present, the use of illicit drugs in the last year may lead to more frequent use of e-cigarettes [8]. On the other hand, simultaneous smoking of tobacco and marijuana by mothers in the prenatal period increases the risk of addiction to tobacco products in adolescents, and consequently increases the risk of addiction to e-cigarettes, which proves the intergenerational harm of tobacco smoking.

According to the data presented by Ioakeimidis et al., breastfeeding after childbirth may reduce the risk of returning to smoking, however, the stress associated with the new role for a woman, as well as the presence of smokers in the environment of the child's mother, may result in continuing smoking [4]. If it is desirable to quit traditional cigarettes, e-cigarettes are often chosen then. 


\subsection{Effects of nicotine contained in e-cigarettes and traditional tobacco .}

As it is presented in the study by Peterson et al., the most important biomarkers related to the toxicity and carcinogenicity of tobacco smoke, allowing to distinguish active from passive smoking, are tobacco-specific compounds: nicotine and its related metabolites, including the carcinogenic metabolite 4-(methylnitrosamine)-1-(3-pyridyl)-1-butanol (NNAL) [7].

It has been proven that the nicotine present in cigarette smoke, passing through the placenta to the fetus, increases the risk of low weight of the child after birth, which depends on the number of cigarettes smoked. Moreover, it may lead to prematurity, intrauterine growth restriction and perinatal death $[4,6,7,13]$. Prenatal exposure to cigarette smoke also causes telomere shortening and contributes to the development of malignant neoplasms in children, type 2 diabetes, asthma, brain development disorders, decreased lung function, and increased susceptibility to more frequent respiratory infections in children [5,7].

Nicotine, also present in most e-cigarettes, has the greatest negative impact on lung development among all components of tobacco smoke. The maternal smoking exposure is associated with the pathogenesis of asthma in children [2,3]. Studies in mice show that during pregnancy, nicotine stimulates branched airway morphogenesis, reduces forced expiratory flows, increases airway responsiveness, and increases collagen deposition around the airways. It can additionally cause disorders in the endocrine, respiratory and reproductive systems. Exposure to tobacco smoke in the prenatal period not only affects the perinatal indicators and problems in early childhood, but also increases the later risk of diseases such as obesity, behavioral disorders - hyperactivity, ADHD, problems with concentration, and even cardiovascular diseases [2,7,14]. Poisoning nicotine can occur in organism through ingestion or via the transdermal and transplacental routes.

There is still no information that any dose of nicotine is safe in pregnancy $[1,14]$. Alluding to The USPSTF (United States Preventive Services Task Force) convincing evidence that behavioral interventions substantially improve achievement of tobacco smoking abstinence in pregnant women, increase infant birth weight, and reduce risk for preterm birth [15]. Contrary to common belief that e-cigarettes are less harmful to our health, some studies show that e-cigarettes contain more nicotine than traditional tobacco products. 
2.4 Methods of smoking cessation in pregnancy.

Women are motivated to quit smoking for both their own and fetus health. The studies confirm the beneficial effects of smoking cessation - it results in fewer miscarriages, lower perinatal mortality, and a reduction in the risk of smoking for children in their adolescence [4]. There are several smoking cessation aid methods tested lately in effectiveness and safety for a fetus.

The first method recommended for pregnant women are behavioral interventions [16]. The task of a physician is to present the drawbacks of smoking, especially during pregnancy, following the effective principle of "ask, advise, refer" [15]. Ioakeimidis et al. report that behavioral intervention increases the rate of women quitting smoking, but counseling seems to improve quit rates primarily when combined with pharmacotherapy [4]. Therefore, pharmacotherapy seems to be a useful tool, especially in the case of unsuccessful attempts to quit smoking or for patients who are at high risk for continued smoking - eg. heavy smokers (more than 10 cigarettes per day). Bupropion is considered a very effective option in combating smoking by non-pregnant people, however, along with its metabolites, hydroxybupropion and threohydrobupropion, it can pass through the placenta and cause abnormal fetal development, in particular in the first trimester [4]. Pharmacokinetics of bupropion and varenicline are not fully known, that's why it's difficult to select the appropriate safe and effective doses individually for the patient.

Some studies indicate that the most appropriate pharmacological therapy for the treatment of cigarette addiction is nicotine replacement therapy (NRT) - available in the form of patches or gum, devoid of many toxins compared to tobacco smoke [16]. A result of this therapy was an increase in the chances of quitting smoking by pregnant women by $48 \%$ and a significant decrease in the percentage of pregnant women smoking [4]. In another study NRT used in pregnancy for smoking cessation increases smoking cessation rates measured in late pregnancy by approximately $40 \%$. However, there is evidence, suggesting that when potentially-biased, non-placebo randomised controlled trials are excluded from analyses, NRT is no more effective than placebo [4,16]. The effectiveness of the therapy may also not be entirely satisfactory due to local adverse reactions. Furthermore, the reduction in the effectiveness of the therapy may be caused by the increased nicotine metabolism in pregnancy. 
E-cigarettes are one of the frequently chosen forms of smoking cessation aid, also by women of childbearing age $[7,8]$ Part of the studies show their effectiveness in combating addiction or reducing cigarettes consumption $[5,6,8,11]$. However, other don't confirm that the use of e-cigarettes by pregnant women helps in smoking cessation [11].

As Lopez et al. and Kurti et al. report, the levels of nicotine and nicotine-related impurities in e-cigarettes vary from brand to brand $[2,6]$. Newer forms of tobacco products reduce the amount of nicotine, which is the main addictive factor, making it easier to quit [2].

According to the FDA, there is currently not enough strong evidence to recommend NRT, e-cigarettes, bupropion or varenicline as an addiction treatment form for pregnant women $[4,11,15]$. Moreover, the WHO warns pregnant women against the use of ecigarettes - the most frequently chosen alternative to traditional cigarettes. [4, 13].

2.5. Potential e-cigarettes' impact on fetus and young children and position of some organizations on smoking e-cigarettes during pregnancy

There are no in vivo, human studies investigating specifically the effects of using ENDS (electronic nicotine delivery systems) during pregnancy on fetus or newborn $[1,4]$. There is however some indirect evidence suggesting how e-cigarettes can influence human prenatal development. Despite the fact that the smoke released from ENDS contains less toxic substances than traditional cigarettes [1], the use of e-cigarettes may still pose a risk to a fetus $[10,12]$.

Nicotine is the most addictive substance of tobacco smoke, also present in ecigarettes, is harmful to the development of the fetus. Although nicotine-free electronic cigarettes are produced, they also contain propylene glycol, fragrances and heavy metals [14].

Propylene glycol is a hazardous substance for pregnant women and children. Under certain conditions, it may result in formation of formaldehyde which is poisonous to them [7]. Thus, exposure to e-cigarette smoke may result in poisoning not only of the mother and fetus, but also of older children, who breath in the smoke. Fragrances can encourage children to inhale the smoke, what in turn often leads to health damage. Additionally, attention is drawn to the possibility of accidentally drinking e-liquid, the effect of which in this form is also assessed as toxic. 
Research conducted so far on animals suggests that e-cigarettes can cause harmful changes in the genome, nervous system or respiratory system disorders [18,19]. However, no harmful effects of e-cigarettes on the fetus have been described in humans. The results of some studies suggest a similar negative effect of nicotine contained in e-cigarettes to that of traditional cigarettes. Also Ioakeimidis in his study does not provide confirmed information on the safety of e-cigarette use in relation to maternal and child health [4]. A similar unknown is the effect of e-cigarettes on fertility, as Kahr et al. notice[17].

The position of health organizations on smoking e-cigarettes during pregnancy seems to be unclear. The FDA has not approved e-cigarettes as an aid in quitting or reducing smoking while other organizations indicate their use as second-line treatment [20]. The WHO also warns pregnant women against using ENDS due to the risk of exposure to abnormal brain development in the fetus and child [16]. According to the USPSTF, there is insufficient evidence for the e-cigarette to be considered an appropriate and safe smoking cessation aid [15].

\section{Summary.}

It seems vital to emphasize the importance of educating young people who are most likely to start smoking in order to prevent the use of all forms of tobacco products - from traditional to e-cigarettes and hookahs, so that they will not endanger the welfare of the fetus as parents.

There is a correlation indicating that cigarette smoking is much more common in white, less educated women who live below the federal poverty level and have psychiatric disorders.

Most of the work is carried out on a small research group so there is a need for further research on the safety of using e-cigarettes by women before and during pregnancy in order to confirm their impact on female fertility, pregnancy and fetal development.

E-cigarettes must be examined in terms of its effectiveness as a smoking cessation aid for pregnant women and its impact on maternal health and the health of the fetus. There is also a high need to determine how long it is safe to use e-cigarettes for pregnant women.

Doctors should be aware of the benefits and risks of using e-cigarettes as an alternative to traditional cigarettes when trying to quit smoking by pregnant women. 


\section{References}

1 Bhandari NR, Day KD, Payakachat N. Use and risk perception of electronic nicotine delivery systems and tobacco in pregnancy. Womens Health Issues. 2018;28: 251-257. doi:10.1016/j.whi.2018.02.005.

2 Lopez AA, Redner R, Kurti AN, Keith DR, Villanti AC, Stanton CA, Gaalema DE, Bunn JY, Doogan NJ, Cepeda-Benito A, Roberts ME, Higgins ST. Tobacco and nicotine delivery product use in a U.S. national sample of women of reproductive age. Prev Med. 2018;117:61-68. doi:10.1016/j.ypmed.2018.03.001.

3 Farber HJ. The Harm of Tobacco Starts Before Birth. Chest. 2015 Sep;148(3):573-574. doi:10.1378/chest.15-0767.

4 Ioakeimidis N, Vlachopoulos C, Katsi V, Tousoulis D. Smoking cessation strategies in pregnancy: Current concepts and controversies. Hellenic J Cardiol. 2019 Jan Feb;60(1):11-15. doi:10.1016/j.hjc.2018.09.001.

5 Makadia LD, Roper PJ, Andrews JO, Tingen MS. Tobacco Use and Smoke Exposure in Children: New Trends, Harm, and Strategies to Improve Health Outcomes. Curr Allergy Asthma Rep. 2017 Aug;17(8):55. doi:10.1007/s11882-017-0723-0.

6 Kurti AN, Redner R, Lopez AA, Keith DR, Villanti AC, Stanton CA, Gaalema DE, Bunn JY, Doogan NJ, Cepeda-Benito A, Roberts ME, Phillips J, Higgins ST. Tobacco and nicotine delivery product use in a national sample of pregnant women. Prev Med. 2017;104:50-56. doi:10.1016/j.ypmed.2017.07.030.

7 Peterson LA, Hecht SS. Tobacco, e-cigarettes, and child health. Curr Opin Pediatr. 2017;29(2):225-230. doi:10.1097/MOP.0000000000000456.

8 De Genna NM, Richardson GA, Goldschmidt L, Day NL, Cornelius MD. Prenatal exposures to tobacco and cannabis: Associations with adult electronic cigarette use. Drug Alcohol Depend. 2018;188:209-215. doi:10.1016/j.drugalcdep.2018.03.045.

9 Wigginton B, Gartner C, Rowlands IJ. Is it safe to vape? Analyzing online forums discussing e-cigarette use during pregnancy. Womens Health Issues. 2017;27(1): 93-99. doi: 10.1016/j.whi.2016.09.008.

10 Schilling L, Schneider S, Maul H, Spallek J. Study on E-Cigarettes and Pregnancy (STEP) - Study Protocol of a Mixed Methods Study on Risk Perception of E-Cigarette Use During Pregnancy and Sample Description. Geburtshilfe Frauenheilkd. 2020;80(1): 66-75. doi:10.1055/a-1061-7288.

11 Chiang SC, Abroms LC, Cleary SD, Pant I, Doherty L, Krishnan N. E-cigarettes and smoking cessation: a prospective study of a national sample of pregnant smokers. BMC Public Health. 2019;19(1):964. Published 2019 Jul 18. doi:10.1186/s12889-019-7299-7.

12 Kapaya M, D'Angelo DV, Tong VT, England L, Ruffo N, Cox S, Warner L, Bombard J, Guthrie T, Lampkins A, King BA. Use of Electronic Vapor Products Before, During, and After Pregnancy Among Women with a Recent Live Birth - Oklahoma and Texas, 2015. MMWR Morb Mortal Wkly Rep. 2019;68(8): 189-194. doi:10.15585/mmwr.mm6808a1.

13 Spindel ER, McEvoy CT. The Role of Nicotine in the Effects of Maternal Smoking during Pregnancy on Lung Development and Childhood Respiratory Disease. Implications for Dangers of E-Cigarettes. Am J Respir Crit Care Med. 2016;193(5): 486494. doi:10.1164/rccm.201510-2013PP. 
14 Wong MK, Barra NG, Alfaidy N, Hardy DB, Holloway AC. Adverse effects of perinatal nicotine exposure on reproductive outcomes. Reproduction. 2015 Dec;150(6):R185-93. doi:10.1530/REP-15-0295.

15 Siu AL. U.S. Preventive Services Task Force, Behavioral and Pharmacotherapy Interventions for Tobacco Smoking Cessation in Adults, Including Pregnant Women: U.S. Preventive Services Task Force Recommendation Statement. Ann Intern Med. 2015 Oct 20;163(8):622-34. doi:10.7326/M15-2023.

16 Coleman T, Chamberlain C, Davey MA, Cooper SE, Leonardi-Bee J. Pharmacological interventions for promoting smoking cessation during pregnancy. Cochrane Database Syst Rev. 2015 Dec 22;(12):CD010078. doi:10.1002/14651858.CD010078.pub2.

17 Kahr MK, Padgett S, Shope CD, Griffin EN, Xie SS, Gonzalez PJ, Levison J, Mastrobattista J, Abramovici AR, Northrup TF, Stotts AL, Aagaard KM, Suter MA. A qualitative assessment of the perceived risks of electronic cigarette and hookah use in pregnancy. BMC Public Health. 2015; 15: 1273. doi:10.1186/s12889-015-2586-4.

18 Chen H, Li G, Chan YL, Chapman DG, Sukjamnong S, Nguyen T, Annissa T, McGrath KC, Sharma P, Oliver BG. Maternal E-Cigarette Exposure in Mice Alters DNA Methylation and Lung Cytokine Expression in Offspring. Am J Respir Cell Mol Biol. 2018 Mar;58(3):366-377. doi: 10.1165/rcmb.2017-0206RC.

19 Nguyen T, Li GE, Chen H, Cranfield CG, McGrath KC, Gorrie CA. Maternal E-Cigarette Exposure Results in Cognitive and Epigenetic Alterations in Offspring in a Mouse Model. Chem Res Toxicol. 2018 Jul 16; 31(7):601-611. doi: 10.1021/acs.chemrestox.8b00084.

20 U. S. Food and Drug Administration. Section 1 of the Tobacco Control Act - Public Law 111-131 [H.R. 1256] [Available from: https://www.fda.gov/TobaccoProducts/Labeling/RulesRegulationsGuidance/ucm261829. htm. 